05

\title{
Влияние неопределенности значений вероятностей спонтанного излучения на температурное поведение коэффициентов ударного самоуширения спектральных линий молекул $\mathrm{CO}_{2}$
}

\author{
() К.И. Аршинов , В.В. Бобровский, О.Н. Крапивная, В.Н. Шут \\ Институт технической акустики НАН Беларуси, \\ 210009 Витебск, Белоруссия \\ e-mail: arshinov.k@yandex.ru
}

Поступила в редакцию 18.05.2020 г.

В окончательной редакции 18.05.2020 г.

Принята к публикации 16.06.2020 г.

\begin{abstract}
Изучено влияние отклонения значений вероятностей спонтанного излучения $A$ на температурное поведение коэффициентов ударного самоуширения $\gamma(T)$ спектральных линий молекул $\mathrm{CO}_{2}$ при решении обратной задачи совместного определения $A$ и $\gamma$ из массива коэффициентов поглощения. Исследования проведены для двух случаев: 1) в расчетах использовали $A=0.2022 \mathrm{~s}^{-1}$ (HITRAN2012), 2) значения $A$ были получены при минимизации суммы взвешенных квадратов отклонений коэффициентов поглощения. Для двух вариантов расчета установлено, что при измерениях коэффициентов поглощения с погрешностью не более $0.05 \alpha_{\min }$ отклонение функций $\gamma(T)$ не превышает $5 \%$ в диапазоне температур $300-700 \mathrm{~K}$. Причинноследственной связи между параметрами $\gamma$ и $A$ не наблюдается. Метод наименьших квадратов позволяет при совместном определении параметров $\gamma(T)$ и $A$ корректно рассчитывать температурные зависимости коэффициентов ударного самоуширения линий $\mathrm{CO}_{2}$.
\end{abstract}

Ключевые слова: диоксид углерода, коэффициент ударного самоуширения, вероятность спонтанного излучения, корреляция.

DOI: $10.21883 /$ OS.2020.10.50024.152-20

\section{1. Введение}

Вид температурных зависимостей столкновительных ширин линий поглощения различных переходов молекул $\mathrm{CO}_{2}$ необходимо знать при расчетах переноса излучения в атмосфере и проектировании мощных $\mathrm{CO}_{2}$-лазеров, а также при использовании перестраиваемых $\mathrm{CO}_{2}$-лазеров в методах многочастотной диагностики атмосферы и нагретых газообразных продуктов сгорания. Поэтому экспериментальному определению вероятностей спонтанного излучения $A$ ( $A-$ коэффициенты Эйнштейна) и коэффициентов ударного самоуширения спектральных линий $\mathrm{CO}_{2}$ посвящено достаточно большое число работ [1-8]. Тем не менее вопросы, касающиеся экспериментального определения температурной зависимости коэффициента ударного самоуширения, нельзя считать полностью решенными.

Из анализа работ, относящихся к экспериментальному определению $\gamma$ и $A$ для линии $P(20)$ перехода $10^{0} 0-00^{0} 1 \mathrm{CO}_{2}$ при $T=296 \mathrm{~K}$, следует, что значения $\gamma$ находятся в диапазоне $6.5-10.3 \mathrm{MHz} / \mathrm{Torr}$, а значения $A$ - в диапазоне $0.15-0.32 \mathrm{~s}^{-1}[9]$. В $[10,11]$ отмечается, что показатель степени $n$ функции зависит от выбранного температурного диапазона и предлагается рассматривать несколько зависимостей типа $\gamma(t)=\gamma\left(T_{0}\right)\left(T_{0} / T\right)^{n}$, но не объясняются причины получения разных $n$ и $T_{0}$. Относительные коэффициенты ударного уширения линий $\mathrm{CO}_{2}$ буферными газами $\beta_{\mathrm{CO}_{2}-M_{j}}(T)$ также отличают- ся. В [11-13], например, для линий $R 22$ и $R 34$ перехода $00^{0} 0-22^{0} 1$ с ростом температуры функции $\beta_{\mathrm{CO}_{2}-M_{j}}(T)$ имеют слабо спадающий вид как для $\mathrm{N}_{2}$, так и $\mathrm{O}_{2}$. В [14] для этих линий получены разнонаправленные температурные зависимости $\beta_{\mathrm{CO}_{2}-\mathrm{N}_{2}}(T)$ и $\beta_{\mathrm{CO}_{2}-\mathrm{O}_{2}}(T)$.

Использование стабилизированного по максимуму контура усиления перестраиваемого $\mathrm{CO}_{2}$-лазера в качестве источника зондирующего излучения упрощает задачу экспериментального определения параметров $A$ и $\gamma$ линий переходов $10^{0} 0-00^{0} 1$ и $02^{0} 0-00^{0} 1$.

Известны различные методы определения коэффициентов ударного самоуширения спектральных линий $\mathrm{CO}_{2}$ - гетеродина $[15,16]$, абсорбции излучения перестраиваемого диодного лазера по всему контуру линии [17], резонансного поглощения в центре линий с лоренцевым профилем, сформированным столкновениями молекул [18]. В [19] искомый параметр $\gamma$ рассчитывался из отношения коэффициента поглощения (КП) с лоренцевым и допплеровским контурами линий. В [5,7,14,1821] использовался метод наименьших квадратов (МНК), который является одним из статистических методов, позволяющих, в частности для нашей задачи, совместно определить параметры $A$ и $\gamma$. Применение принципа максимального правдоподобия при нормальном законе распределения ошибок приводит к вычислительной схеме МНК, которая позволяет получать состоятельные, несмещенные и эффективные оценки [22]. 
Цель данной работы состоит в выяснении влияния неопределенности значений вероятностей спонтанного излучения $A$ на температурное поведение коэффициентов ударного самоуширения $\gamma(T)$ спектральных линий молекул $\mathrm{CO}_{2}$ при решении обратной задачи совместного определения $A$ и $\gamma$ с использованием массива коэффициентов поглощения с фойгтовским профилем линий.

\section{2. Методика}

Bсе расчеты проведены для линии $R 22$ перехода $10^{0} 0-00^{0} 1$ молекулы $\mathrm{CO}_{2}$. Для фиксированной температуры записывается фундаментальная система уравнений

$$
\hat{\alpha}_{j}=\alpha_{j}(A, \gamma)+\Delta_{j},
$$

где $\hat{\alpha}_{j}$ - значение КП при давлении $p_{j}, \alpha_{j}(A, \gamma)-$ функция, описывающая КП, $\Delta_{j}-$ погрешность (невязка) $j$-го измерения.

Выражение для КП на центральной частоте $v_{0}$ любой линии перехода $10^{0} 0-00^{0} 1$ молекулы $\mathrm{CO}_{2}$ имеет вид

$$
\alpha_{i f}\left(v_{0}\right)=\frac{c^{2}}{8 p i v_{0}^{2}} N_{\mathrm{CO}_{2}} A_{f i} g_{f} Q_{V}^{-1} Q_{R f}^{-1}\left(e^{-\frac{E_{i}}{k T}}-e^{-\frac{E_{f}}{k T}}\right) F_{i f}\left(v_{0}\right),
$$

где индекс $i$ соответствует нижнему уровню $10^{0} 0$ с энергией $E_{i}=\omega_{100}+B_{100} J_{i}\left(J_{i}+1\right)$, индекс $f-$ верхнему уровню $00^{0} 1$ с энергией $E_{f}=\omega_{100}+B_{001} J_{f}\left(J_{f}+1\right)$, $\omega_{100}=1388.3 \mathrm{~cm}^{-1}$ и $\omega_{001}=2349.3 \mathrm{~cm}^{-1}$ — энергии, $B_{100}=0.39018783 \mathrm{~cm}^{-1}$ и $B_{001}=0.38714044 \mathrm{~cm}^{-1}$ вращательные постоянные рассматриваемых уровней [23], $\mathrm{N}_{\mathrm{CO}_{2}}$ - концентрация молекул $\mathrm{CO}_{2}, A_{f i}-$ вероятность спонтанного излучения, $g_{f}=2 J_{f}+1-$ вырождение уровня $f$,

$$
Q_{R f}=\left(2 h c B_{f} / k T\right)^{-1}
$$

— вращательная,

$$
\begin{aligned}
& Q_{v}=\left[1-\exp \left(-\frac{1997 \mathrm{~K}}{T}\right)\right]^{-1} \\
& \times\left[1-\exp \left(-\frac{960 \mathrm{~K}}{T}\right)\right]^{-2}\left[1-\exp \left(-\frac{3380 \mathrm{~K}}{T}\right)\right]^{-1}
\end{aligned}
$$

- колебательная статистические суммы молекулы $\mathrm{CO}_{2}$, $F_{i f}\left(v_{0}\right)$ - формфактор в центре $J$-й линии поглощения $[24,25]$.

Аналитическое выражение для фойгтовского формфактора в центре линии (модуль относительной ошибки менее $0.9 \%$ ) имеет [26] вид

$F_{i f}\left(v_{0}\right)=\left[\Delta v_{L}+\sqrt{\left(\frac{\pi-2}{2}\right)^{2}\left(\Delta v_{L}\right)^{2}+\frac{\pi}{4 \ln (2)}\left(\Delta v_{D}\right)^{2}}\right]^{-1}$,

где

$$
\Delta v_{D}=\frac{2 v_{0}}{c} \sqrt{\frac{2 \ln (2) k T}{M}}
$$

- допплеровская ширина линии поглощения (на полувысоте), $M$ - масса молекулы $\mathrm{CO}_{2}, \Delta v_{L}=\gamma(T) p$ лоренцева ширина линии поглощения.

МНК-оценки $(A, \gamma)$ обращают в минимум сумму взвешенных квадратов невязок, полученную из системы фундаментальных уравнений (1):

$$
Z=\sum_{j} w_{j}\left[\hat{\alpha}_{j}-\alpha_{j}(A, \gamma)\right]^{2}
$$

где $w_{j}-$ весовой коэффициент $j$-го измерения. Наиболее употребляемыми способами минимизации функционала (4) являются перебор значений аргументов и градиентные методы. Из выражений (2) и (3) видно, что уравнения фундаментальной системы (1) линейные относительно $A$ и нелинейные для $\gamma$. Минимизация осуществлялась путем численного сканирования коэффициента ударного самоуширения $\gamma$ с последующим расчетом вероятности спонтанного излучения $A$ согласно выражению

$$
A=\frac{\sum_{j} w_{j} \hat{\alpha}_{j} \varphi_{j}}{\sum_{j} w_{j}\left(\varphi_{j}\right)^{2}}
$$

которое получено из условия $\frac{\partial Z}{\partial A}=0$, где $\varphi_{j}=\frac{\partial \alpha_{j}}{\partial A_{f i}}$.

Таким образом, выбирается пара параметров $(A, \gamma)$, которой соответствует минимальное значение $Z$. Для первой итерации брались весовые коэффициенты $w_{j}^{(1)}=1$, а для $(u+1)$-й весовые коэффициенты [27]

$$
w_{j}^{(u+1)}=\frac{1}{\left[\hat{\alpha}_{j}-\alpha_{j}\left(A^{(u)}, \gamma^{(u)}\right)\right]^{2}} .
$$

Погрешности искомых параметров определялись путем расчета ковариационных матриц [27,28]. При прямых некоррелированных измерениях КП $\hat{\alpha}_{j}$ с одинаковым среднеквадратичным отклонением (СКО) $\sigma_{\alpha}$ диагональные элементы ковариационной матрицы представляют собой дисперсии искомых параметров. Связь между ковариационными матрицами, прямо $\left(\hat{\alpha}_{j}\right)$ и косвенно $(A, \gamma)$ определяемыми параметрами может быть записана в виде

$$
C O V=\sigma_{\alpha}^{2}\left(b^{t} b\right)^{-1},
$$

где $b$ - матрица с элементами $b_{j l}=\frac{\partial \alpha_{j}}{\partial y_{l}} \quad(l=1,2$; $\left.y_{1}=A, y_{2}=\gamma\right)$ в точке квазирешения, $b^{t}-$ транспонированная матрица $b$. Выражение (5) связывает дисперсии искомых параметров $\sigma_{A}^{2}, \sigma_{\gamma}^{2}$ и исходных данных $\sigma_{\alpha}^{2}($ СКО $\sigma_{\alpha}$ не более $2 \cdot 10^{-5} \mathrm{~cm}^{-1}$ ).

\section{3. Результаты}

Решение поставленной задачи осуществлялось поэтапно. На первом этапе, используя ранее экспериментально полученные значения КП для линии $R 22$ [21], методом наименьших квадратов были определены значения коэффициентов $\gamma$ и $A$ в диапазоне $300-700 \mathrm{~K}$. На втором этапе, используя полученную температурную зависимость 
Измеренные значения коэффициентов $\gamma$ и $A$ линии $R 22$ перехода $10^{0} 0-00^{0} 1$ молекулы $\mathrm{CO}_{2}$ в диапазоне $300-700 \mathrm{~K}[21]$

\begin{tabular}{l|c|c|c|c|c|c|c|c|c|c|c}
\hline$T, \mathrm{~K}$ & 300 & 310 & 320 & 330 & 350 & 380 & 400 & 450 & 500 & 600 & 700 \\
\hline$\gamma, \mathrm{MHz} /$ Torr & 10.76 & 10.44 & 10.45 & 10.08 & 9.069 & 8.04 & 7.501 & 6.556 & 5.668 & 4.803 & 4.7 \\
\hline$A, \mathrm{~s}^{-1}$ & 0.2783 & 0.3217 & 0.3540 & 0.3729 & 0.3806 & 0.3734 & 0.3558 & 0.3230 & 0.2775 & 0.2394 & 0.2350
\end{tabular}

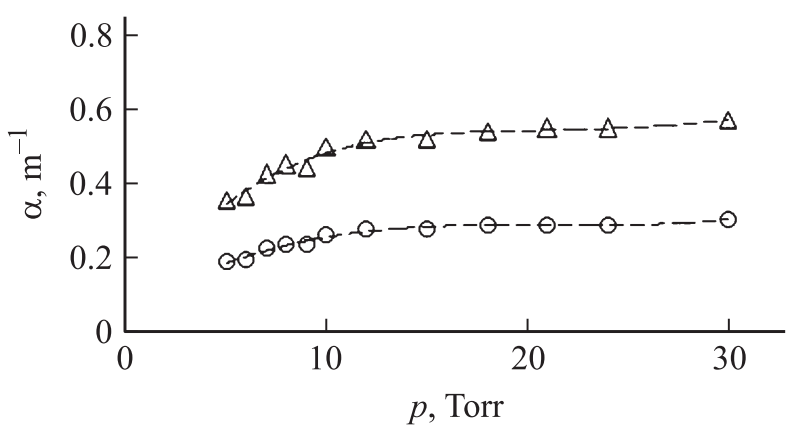

Рис. 1. Зависимость рассчитанных коэффициентов поглощения $\alpha_{j}$ (с наложением гауссова шума $0.05 \alpha_{\min }$ ) от давления чистого $\mathrm{CO}_{2}$ для линии $R 22$ : $\circ-A=0.2022 \mathrm{~s}^{-1}$ (HITRAN2012), $\Delta-A=0.2783 \mathrm{~s}^{-1}$ (таблица), $T=300 \mathrm{~K}$.

$\gamma(T)$ и различающиеся значения $A$, по формуле (2) были рассчитаны КП, на которые в дальнейшем наносился шум. Сравнивались два варианта $-A=0.2022 \mathrm{~s}^{-1}$ во всем температурном диапазоне и разные значения $A$, полученные в [21]. Таким образом, на втором этапе подготовлены необходимые для дальнейших расчетов исходные данные (КП) с заранее известными температурными зависимостями изучаемых параметров. На третьем этапе для двух вариантов методом наименьших квадратов совместно определялись значения $A$ и $\gamma$ во всем температурном диапазоне. Затем проведены сравнения температурных зависимостей $\gamma(T)$, полученных при разных значениях $A$.

Предварительно был исследован вид функции распределения ошибок КП. Измерения КП в каждой рабочей точке содержали выборку $\left(\alpha_{1}, \alpha_{2}, \ldots \alpha_{j}\right)$, где $j=100$. Вид построенной гистограммы позволял допустить, что функция плотности вероятностей имеет нормальное распределение. Для предварительной оценки гипотезы о нормальном распределении ошибок были рассчитаны коэффициенты асимметрии и эксцесса. Значения коэффициентов асимметрии и эксцесса были в два раза меньше их среднеквадратичных ошибок, что соответствует нормальному распределению. Кроме того, для проверки согласия эмпирической функции распределения с теоретической модельной функцией рассматривались основные критерии согласия: $\chi^{2}-$ критерий Пирсона и $\lambda$ - критерий Колмогорова. Расчеты показали, что предположение о нормальном распределении ошибок измеренных КП правомерно [22,29].
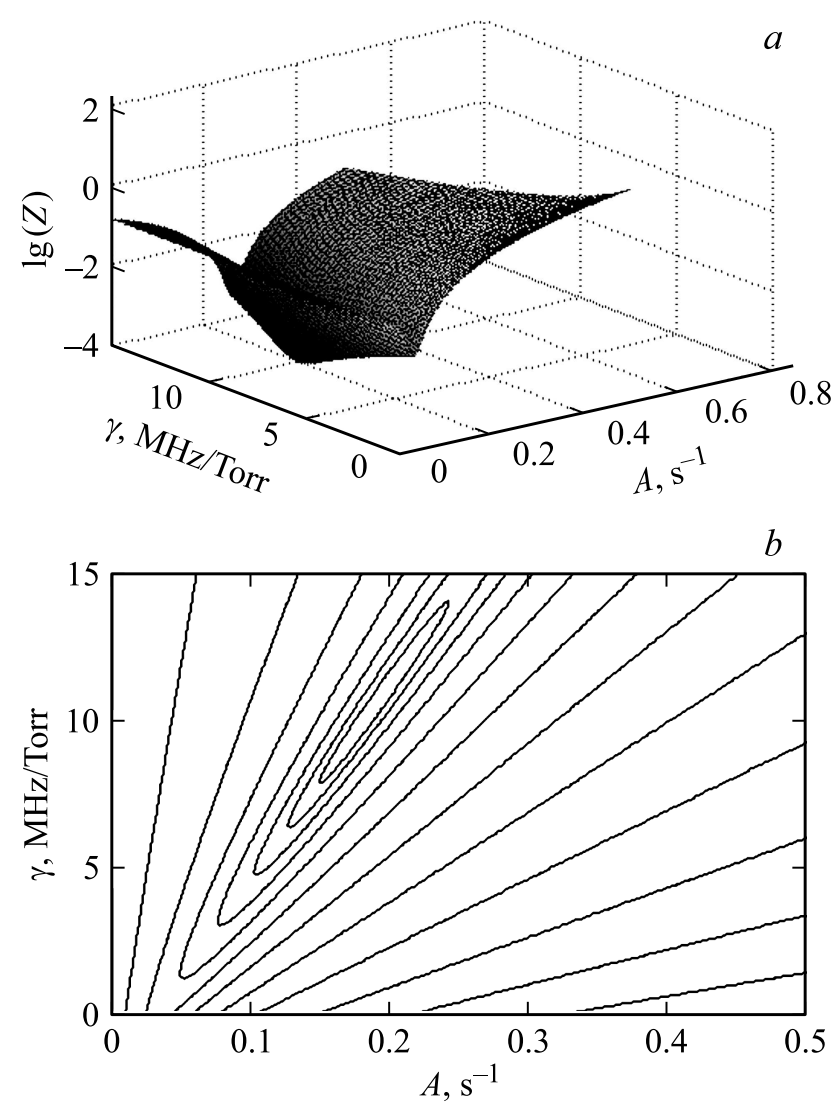

Рис. 2. (a) Функционал $\lg (Z)=f(A, \gamma),(b)$ контуры сечений функционала $\lg (Z)$.

\section{Этап I}

В работе [21] представлены результаты экспериментальных измерений КП, которые были проведены в диапазоне давлений 5-30 Torr, когда контур линий поглощения описывается функцией Фойгта. На основе измеренных КП в диапазоне температур 300-700 К были совместно найдены вероятности спонтанного излучения и коэффициенты ударного самоуширения для линии $R 22$ перехода $10^{0} 0-00^{0} 1 \mathrm{CO}_{2}$ (таблица). Принимая во внимание близость значений температур и давлений поглощающего газа с условиями тлеющего разряда в отпаянном электроразрядном $\mathrm{CO}_{2}$-лазере, можно считать, что измерения были выполнены на центральных частотах линий поглощения. Искомые параметры рассчитывались методом наименьших квадратов. 
Установлено, что температурная зависимость коэффициента ударного самоуширения имеет следующий вид:

$$
\gamma(T)=\gamma_{c}\left(T_{c} / T\right)^{n},
$$

где $T_{c}=463 \mathrm{~K}, \gamma_{c}=6.315 \mathrm{MHz} / \mathrm{Torr}, n=n_{1}=1.361$ при $T<T_{c}, n=n_{2}=0.887$ при $T>T_{c}$.

\section{Этап II}

Для выяснения степени влияния $A$ на $\gamma$ были рассчитаны зависимости КП от давления в диапазоне 5-30 Torr при некоторой фиксированной температуре в соответствии с выражениями (2) и (3) с наложением гауссова шума $\left(0.05 \alpha_{\min }\right)$. Расчеты проведены для двух случаев: 1) вероятность спонтанного излучения имеет постоянную величину — для линии $R 22 A=0.2022 \mathrm{~s}^{-1}$ HITRAN2012 [4], 2) вероятность принимает значения, представленные в таблице.

В качестве примера на рис. 1 даны зависимости КП от давления при $A=0.2022 \mathrm{~s}^{-1}$ и $A=0.2783 \mathrm{~s}^{-1}$ (таблица) при температуре $T=300 \mathrm{~K}$. Такие зависимости использовались в качестве исходных данных для определения $\gamma$ и $A$. Видно, что значение коэффициента $A$ оказывает заметное влияние на величины КП.

Далее в среде Matlab была проведена верификация минимума суммы взвешенных квадратов невязок $Z$ (4). Результаты анализа для случая $A=0.2022 \mathrm{~s}^{-1}$ и $T=300 \mathrm{~K}$ представлены на рис. 2. Видно, что имеется глобальный минимум, который наблюдается для следующих значений параметров: $\gamma \approx 11.5 \mathrm{MHz} /$ Torr, $A \approx 0.2 \mathrm{~s}^{-1}$.

Основными механизмами уширения линий в рассматриваемых областях давлений и температур являются эффект Допплера и соударение молекул. Наиболее сложный вид имеют контуры линий, когда ширины допплеровской и лоренцевой составляющих сопоставимы. Простейшей моделью контура, учитывающей оба упомянутых механизма уширения, является контур Фойгта свертка гауссовой и лоренцевой функций (DL/VGT). В ряде работ было обнаружено, что контур DL/VGT не способен описать наблюдаемую форму линий в пределах погрешности измерений [30]. Искажение контура Фойгта происходит из-за соударений с изменением скорости поглощающих молекул (эффект Дике и эффект ветра), которые оказывают влияние на допплеровскую и лоренцеву составляющие контуров [25,30-32]. Поэтому следует оценить влияние значений формфактора контура Фойгта в центре линий на точность решения обратной задачи. В $[25,30]$ приведены оценки эффектов Дике и ветра для молекулы воды. Ширина лоренцевой составляющей контура Фойгта, учитывающей эффект ветра $\Delta_{\mathrm{L} / \mathrm{SDV}}$ (speed dependence Voight), на $\sim 6 \%$ больше соответствующей составляющей $\Delta_{\mathrm{VL} / \mathrm{V}}$ для обычных контуров Фойгта [30]. Эффект Дике приводит к увеличению высоты контура молекул $\mathrm{H}_{2} \mathrm{O}$ на $\sim 8 \%$ [25], и, следовательно, допплеровская составляющая контура сузится. Предположим, что для линии $R 22 \mathrm{CO}_{2}$ из-за упомянутых эффектов искажение составляющих контура того же порядка, что и для $\mathrm{H}_{2} \mathrm{O}$. Решение обратной задачи дает оценку $\gamma$, которая позволяет получить значение КП с погрешностью менее $1 / 5 \sigma_{\alpha}$. Поэтому в нашей работе упомянутые эффекты не учитывались, и в расчетах использовалось аналитическое выражение (3) для традиционного контура Фойгта [26].

Совместное определение параметров $(A, \gamma)$ по сути представляет собой процесс получения двумерных случайных величин $(\mathrm{CB})(A, \gamma)$. Связь, которая существует между СВ разной природы, например, между СВ $\gamma$ и $\mathrm{CB} A$, не обязательно является следствием прямой зависимости одной величины от другой, так называемой функциональной связи. В некоторых случаях обе СВ зависят от целой совокупности разных факторов, общих для обеих величин, в результате чего и формируются связанные друг с другом закономерности. Когда связь между СВ обнаружена с помощью статистики, нельзя утверждать, что обнаружена причина происходящего изменения параметров, возможно скорее проявились два взаимосвязанных следствия. В нашем случае спектральный КП $\alpha$ в центре линии $\mathrm{CO}_{2}$ является $\mathrm{CB}$, описывающей характеристику рассматриваемого физического объекта (нагретый диоксид углерода в диапазоне температур $300-700 \mathrm{~K}$ и диапазоне давлений 5-30 Torr). Выше упоминалось, что функция плотности вероятностей для КП имеет нормальное распределение. Известно, что если двумерная $\mathrm{CB} \alpha(A, \gamma)$ имеет нормальное распределение погрешностей, то аналогичные распределения имеют $\mathrm{CB}(A)$ и $\mathrm{CB}(\gamma)$. На рис. 3, $a$ приведен массив из 35 квазирешений обратной задачи для линии $R 22$ перехода $10^{0} 0-00^{0} 1 \mathrm{CO}_{2}$ при фиксированной температуре $T=300 \mathrm{~K}$. Для определения уровня взаимосвязи между искомыми параметрами $\gamma$ и $A$ в Excel с помощью встроенной функции „КОРЕЛЛ“ был рассчитан коэффициент корреляции $r=0.981$. Тем не менее наличие корреляции не означает наличия функциональной (причинно-следственной) связи между параметрами $\gamma$ и $A$ [33]. Корреляция может быть случайной и объясняться иными факторами, оставшимися за рамками анализа, т.е. причинно-следственные связи порождают корреляцию, но корреляция не означает наличия причинно-следственных связей. В этом случае необходимо дополнительное изучения возможности возникновения взаимной связи, в частности, между параметрами $\gamma$ и $A$, поэтому было рассмотрено получение выражения для КП (см. Приложение). Коэффициент ударного самоуширения $\gamma$ содержится в формфакторе $F\left(v_{0}\right)$, в то время как параметр $A$ используется при описании поглощения и испускания излучения. Следовательно, при получении (2) функциональной связи между параметрами $\gamma$ и $A$ не было установлено.

Принято считать, что условию $0<r<0.2$ соответствует очень слабая связь между случайными величинами. Далее были определены коэффициенты корреляции между параметрами $A$ и $\gamma_{c}, T_{c}, n_{1}: r\left(A, \gamma_{c}\right)=-0.045$, $r\left(A, T_{c}\right)=0.342, r\left(A, n_{1}\right)=0.184$ (рис. $\left.3, b, c, d\right)$. То есть 

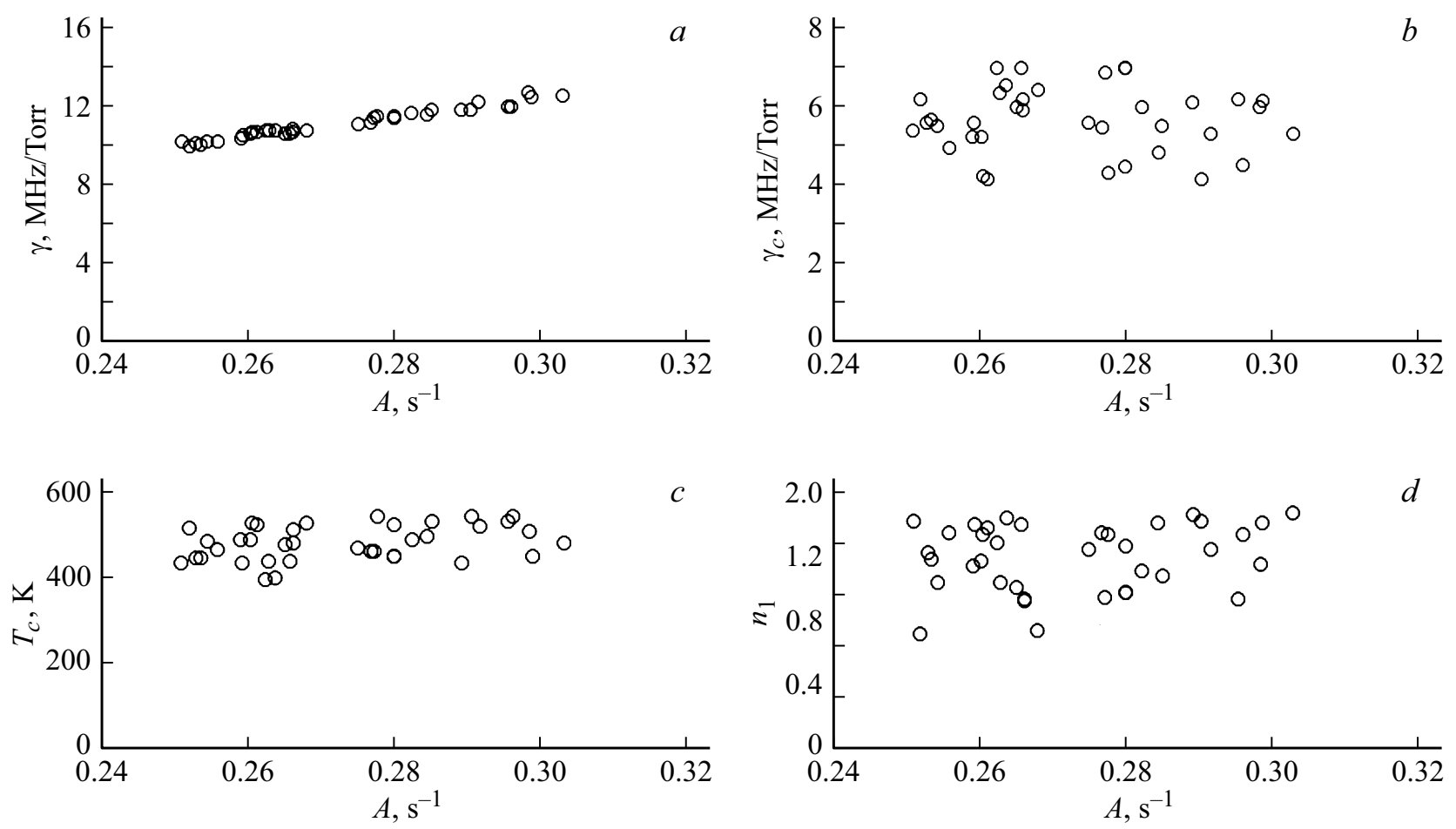

Рис. 3. Массив квазирешений обратной задачи совместного определения коэффициента ударного самоуширения $\gamma$ и вероятности спонтанного излучения $A$ для линии $R 22$ перехода $10^{0} 0-00^{0} 1 \mathrm{CO}_{2}$ при температуре $T=300 \mathrm{~K}: r(A, \gamma)=0.981(a)$, $r\left(A, \gamma_{c}\right)=-0.045(b), r\left(A, T_{c}\right)=0.342(c), r\left(A, n_{1}\right)=0.184(d)$.

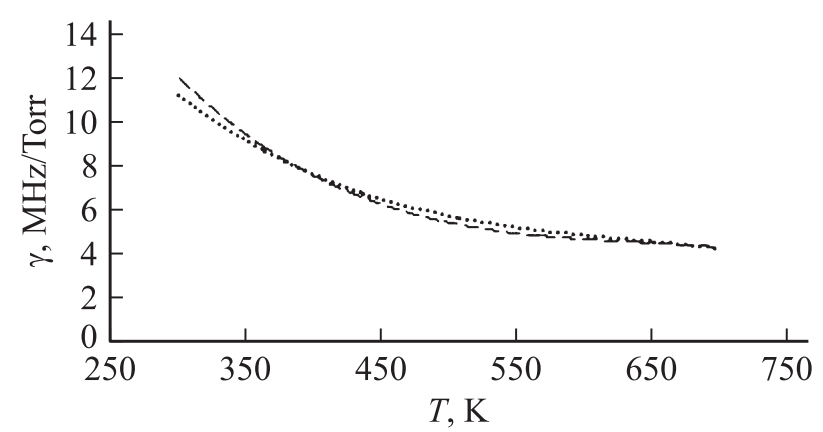

Pис. 4. Температурные зависимости коэффициентов ударного самоуширения для линии $R 22$ перехода $10^{0} 0-00^{0} 1 \mathrm{CO}_{2}$ в диапазоне $300-700 \mathrm{~K}$, полученные при решении обратной задачи: СКО КП $\sigma_{\alpha}=0.05 \alpha_{\min },(----) A$ из таблицы, (---) $A=0.2022 \mathrm{~s}^{-1}$.

связь между вероятностью $A$ и константами, определяющими температурное поведение коэффициента ударного самоуширения, является слабой.

\section{Этап III}

Методом наименьших квадратов осуществлен совместный расчет искомых параметров $\left(\gamma_{c}, A, T_{c}, n_{1}, n_{2}\right)$. Результаты решения обратной задачи для коэффициента ударного самоуширения $\gamma$ представлены на рис. 4 . На рис. 4 видно, что для двух вариантов расчета имеет место практически полное совпадение функций $\gamma(T)$.
Аппроксимации полученных зависимостей могут быть представлены в виде $\gamma(T)=\gamma_{c}\left(T_{c} / T\right)^{n}$ :

1) для $A$ из таблицы $\gamma_{c}=5.834 \pm 1 \mathrm{MHz} /$ Torr, $T_{c}=$ $=492 \pm 49 \mathrm{~K}, n=n_{1}=1.33 \pm 0.006$ при $T<T_{c}, n=$ $=n_{2}=0.873 \pm 0.038$ при $T>T_{c}$;

2) для $A=0.2022 \mathrm{~s}^{-1} \gamma_{c}=5.352 \pm 1 \mathrm{MHz} /$ Torr, $T_{c}=$ $=502 \pm 49 \mathrm{~K}, n=n_{1}=1.572 \pm 0.006$ при $T<T_{c}, n=$ $=n_{2}=0.633 \pm 0.038$ при $T>T_{c}$.

Данные рис. 4 позволяют утверждать, что в диапазоне $300-700 \mathrm{~K}$ параметры $\gamma$ и $A$ не имеют взаимной зависимости. При получении выражения для ненасыщенного коэффициента поглощения не обнаружено функциональной связи между $\gamma$ и $A$. Следовательно, МНК позволяет корректно определять температурные зависимости коэффициентов ударного самоуширения. Вероятности спонтанного излучения линейно входят в выражение КП (2) и оказывают существенное влияния на величину КП, но в силу отсутствия причинно-следственной связи между $\gamma$ и $A$ практически не влияют на вид функции $\gamma(T)$.

\section{Заключение}

Методом наименьших квадратов определены температурные зависимости коэффициентов ударного самоуширения для линии $R 22$ перехода $10^{0} 0-00^{0} 1 \mathrm{CO}_{2}$ в температурном диапазоне $300-700 \mathrm{~K}$ для двух случаев. В первом случае использовалась вероятность спонтанного излучения $A=0.2022 \mathrm{~s}^{-1}$ (HITRAN2012). Во втором 
случае значения $A$ получены при минимизации суммы взвешенных квадратов отклонений коэффициентов поглощения. Установлено, что во всем диапазоне температур 300-700 К для двух вариантов расчета имеет место практически полное совпадение функций $\gamma(T)$ (отклонение не более 5\% при погрешности измерений КП $\left.0.05 \alpha_{\min }\right)$. Найден коэффициент корреляции между $\gamma$ и $A$ $r=0.981$, но не обнаружена их причинно-следственная связь. На слабую взаимосвязь между рассматриваемыми параметрами указывает совпадение функций $\gamma(T)$, полученных при значительно различающихся вероятностях спонтанного излучения. Следовательно, МНК позволяет корректно рассчитывать температурные зависимости коэффициентов ударного самоуширения линий $\mathrm{CO}_{2}$.

\section{Приложение}

Ненасыщенный коэффициент поглощения для двухуровневой модели $\mathrm{CO}_{2}$-лазера может быть представлен $[34,35]$ в виде

$$
\alpha_{i f}=\frac{h v}{c}\left(N_{i} B_{i f}-n_{f} B_{f i}\right) F\left(v_{0}\right),
$$

где населенности нижнего $i$ и верхнего $f$ уровней [24]

$$
\begin{aligned}
& N_{i}=N_{0} Q_{V}^{-1} Q_{R i}^{-1} g_{i} \exp \left(-E_{i} / k T\right), \\
& N(f)=N_{0} Q_{V}^{-1} Q_{R f}^{-1} g_{f} \exp \left(-E_{f} / k T\right) .
\end{aligned}
$$

В условиях термодинамического равновесия, согласно принципу детального равновесия, можно записать равенство $N_{f}\left\lfloor A_{f i}+B_{f i} \rho(v, T)\right\rfloor=N_{i} B_{i f} \rho(v, T)$, из которого с учетом (2П) получаем выражение для $\rho(v, T)$, где $\rho(v, T)$ - спектральная объемная плотность энергии электромагнитного поля:

$$
\rho(v, T)=\frac{A_{f i}}{B_{f i}}\left[\frac{Q_{R i}^{-1} g_{i} B_{i f}}{Q_{R f}^{-1} g_{f} B_{f i}} \frac{\exp \left(-E_{i} / k T\right)}{\exp \left(E_{f} / k T\right)}-1\right]^{-1}
$$

Сравнивая (3П) с формулой Планка

$$
\rho(v, T)=\frac{8 \pi h v^{3}}{c^{3}}\left[\exp \left(\frac{E_{f}-E_{i}}{k T}\right)-1\right]^{-1},
$$

имеем

$$
\frac{A_{f i}}{B_{f i}}=\frac{8 \pi h v^{3}}{c^{3}}, \quad \frac{Q_{R i}^{-1} g_{i} B_{i f}}{Q_{R f}^{-1} g_{f} B_{f i}}=1 .
$$

Подставляя (2П) в (1П) с учетом (4П), для ненасыщенного КП получаем выражение (2), где $g_{f}=2 J_{f}+1-$ весовой коэффициент вырождения верхнего уровня $00^{0} 1, J_{f}$ - квантовое число верхнего вращательного уровня.

\section{Благодарности}

Авторы выражают благодарность профессору Лешенюку Н.С. за обсуждение статьи и полезные замечания.

\section{Конфликт интересов}

Конфликт интересов у авторов отсутствует.

\section{Список литературы}

[1] Young C., Chapman R.E. // JQSRT. 1974. V. 14. P. 679.

[2] Данилов В.В., Кругляков Э.П., Шунько Е.В. // Журн. прикл. мех. и техн. физ. 1972. № 6. С. 24.

[3] Невдах B.B. // Квант. электрон. 1984. Т. 11. № 8. С. 1622; Nevdakh V.V. // Quant. Electron. 1984. V. 14. N 8. P. 1091.

[4] Rothman L.S., Gordon I.E., Babikov Y., Barbe A., Benner D. Chris, Bernath P.F., Birk M., Bizzocchi L., Boudon V., Brown L.R., Campargue A., Chance K., Cohen E.A., Coudert L.H., Devi V.M., Drouin B.J., Fayt A., Flaud J.-M., Gamache R.R., Harrison J.J., Hartmann J.M., Hill C., Hodges J.T., Jacquemart D., Jolly A., Lamouroux J., Le Roy R.J., Li G., Long D.A., Lyulin O.M., Mackie C.J., Massie S.T., Mikhailenko S., Müller H.S.P., Naumenko O.V., Nikitin A.V., Orphal J., Perevalov V., Perrin A., Polovtseva E.R., Richard C., Smith M.A.H., Starikova E., Sung K., Tashkun S., Tennyson J., Toon G.C., Tyuterev Vl.G., Wagner G. // JQSRT. 2013. V. 130. P. 4.

[5] Аршинов К.И., Лешенюк Н.С. // Квант. электрон. 1995. T. 22. № 3. C. 239; Arshinov K.I., Leshenyuk N.S. // Quant. Electron. 1995. V. 25. N 3. P. 223.

[6] Simeĉková M., Jacquemart D., Rothman L.S., Gamache R.R., Goldman A. // JQSRT. 2006. V. 98. P. 130.

[7] McCubbin T.K., Jr. Mooney T.R. // JQSRT. 1968. V. 8. P. 1255.

[8] Predoi-Cross A., Liu W., Murphy R., Povey C., Gamache R.R., Laraia A.L., McKellar A.R.W., Hurtmans D.R., Malathy Devi V. // JQSRT. 2010. V. 111. P. 1065.

[9] Бирюков А.С., Волков А.Ю., Кудрявиев Е.М., Сериков Р.И. // Квант.электрон. 1976. Т. 3. № 8. С. 1748; Biryukov A.S., Volkov A.Y., Kudryavtsev E.M., Serikov R.I. // Quant. Electron. 1976. V.6. N8. P. 946.

[10] Huang X., Gamache R.R., Freedman R.S., Schwenke D.W., Lee T.J. // JQSRT. 2014. V. 147. P. 134.

[11] Lamouroux J., Gamache R.R., Laraia A.L., Hartmann J.-M., Boulet C. // JQSRT. 2012. V. 113. P. 1536.

[12] Gamache R.R., Lamouroux J., Laraia A.K., Hartmann J.-M., Boulet C. // JQSRT. 2012. V. 113. P. 976.

[13] Gamache R.R., Lamouroux J., Laraia A.K., Hartmann J.-M., Boulet C. // JQSRT. 2012. V. 113. P. 991.

[14] Rosenmann L., Hartmann J.M., Perrin M.Y., Taine J. // Appl. Opt. 1988. V. 27. P. 3902.

[15] Meyer T.W., Rhodes C.K., Haus H.A. // Phys. Rev. A. 1975. V. 12. N 5. P. 1993.

[16] Зеневич С.Г., Климчук А.Ю., Семенов В.М., Спиридонов М.Е., Родин А.В. // Квант. электрон. 2019. Т. 49. № 7. C. 602.

[17] Андреев С.Н., Очкин В.Н., Савинов С.Ю. // Квант. электрон. 2002. T. 32. № 7. C. 647.

[18] Gross L.A., Griffiths P.R. // Appl. Opt. 1987. V. 26. N 11. P. 2250.

[19] Arié E., Lacome N., Levy A. // Appl. Opt. 1987. V. 26. N 9. P. 1636.

[20] Аршинов К.И., Аршинов М.К., Невдах В.В. // Опт. и спектр. 2012. Т. 112. № 6. С. 914; Arshinov K.I., Arshinov M.K., Nevdakh V.V. // Opt. Spectrosc. 2012. V. 112. N 6. P. 844. 
[21] Аршинов К.И., Крапивная О.Н., Невдах В.В., Шут В.Н. // Опт. атм. и океана. 2020. Т. 33. № 1. С. 5; Arshinov K.I., Krapivnaya O.N., Nevdakh V.V., Shut V.N. // Atm. Ocean Opt. 2020. V. 33. N 3. P. 229.

[22] Герасимович А.И. Математическая статистика. Минск: Вышэйшая школа, 1983. $279 \mathrm{c.}$

[23] Bridges T.J., Chang T.Y. // Phys. Rev. Lett. 1969. V. 22. P. 811.

[24] Ачасов О.И., Кудрявцев Н.Н., Новиков С.С., Солоухин Р.И., Фомин Н.А. Диагностика неравновесных состояний в молекулярных лазерах. Минск: Наука и техника, 1985. 208 c.

[25] Быков А.Д., Синица Л.Н., Стариков В.И. Введение в колебательно-вращательную спектроскопию многоатомных молекул. Томск: Изд-во Института оптики атмосферы CO PAH, 2004. 274 c.

[26] Кудря В.П. // ОПт. и спектр. 1983. Т. 55. В. 6. С. 113.

[27] Мудров В.И., Кушко В.Л. Методы обработки измерений. М.: Радио и связь, 1983. 304 с.

[28] Лешенюк Н.С., Пашкевич В.В. // ЖПС. 1987. Т. 46. № 4. C. 567.

[29] Новицкий П.В., Зограф И.А. Оценка погрешностей результатов измерений. Л.: Энергоатомиздат, 1985. 248 с.

[30] Петрова Т.М., Солодов А.М., Щербаков А.П., Дейчули В.М., Солодов А.А., Пономарев Ю.Н., Чеснокова Т.Ю. // Опт. атм. и океана. 2016. Т. 29. № 10. C. 821; Petrova T.M., Solodov A.M., Shcherbakov A.P., Deichuli V.M., Solodov A.A., Ponomarev Yu.N., Chesnokova T.Yu. // Atm. Ocean Opt. 2017. V. 30. P. 123.

[31] Козодоев А.В., Привезенцев А.И., Фазлиев А.З., Филиппов Н.Н. // Опт. атм. и океана. 2017. Т. 30. № 4. С. 329; Kozodoev A.V., Privezentsev A.I., Fazliev A.Z., Filippov N.N. // Atm. Ocean Opt. 2018. V. 31. P. 201.

[32] Раутиан С.Г., Собельман И.И. // УФН. 1966. Т. 90. В. 2. C. 209.

[33] Справочник по вероятностным расчетам. М.: Воениздат, 1970. $536 \mathrm{c}$

[34] Ельяшевич М.А. Атомная и молекулярная спектроскопия. М.: ФМЛ, 1962. 892 c.

[35] Witteman W.J. The $\mathrm{CO}_{2}$ Laser. Berlin, Heidelberg: Springer,1987. Перевод: Виттеман В. $\mathrm{CO}_{2}$-лазер. М.: Мир, 1990. $360 \mathrm{c}$. 